\title{
Strength and stiffness variability study of viscoelastic adhesive butt joints
}

\author{
K. Van Massenhove ${ }^{\mathrm{a}}$ and D. Vandepitte ${ }^{\mathrm{b}}$ and S. Debruyne ${ }^{\mathrm{a}}$ \\ ${ }^{a}$ KU Leuven, Faculty of Engineering Technology, Campus Bruges, Spoorwegstraat 12, 8200 \\ Bruges, Belgium; \\ ${ }^{\mathrm{b}}$ KU Leuven, Faculty of Engineering Science, Celestijnenlaan 300, 3001 Heverlee (Leuven), \\ Belgium
}

\section{ARTICLE HISTORY}

Compiled January 25, 2019

\begin{abstract}
Viscoelastic structural adhesives show great promise in industrial and civil applications where a highly ductile connection between dissimilar components is required, at both low and high load frequencies. Suitable chemistries for these applications are silicones, polyurethanes and MS polymers. These adhesives show the required low modulus and high elongation at joint failure, while retaining satisfactory strength for long term applications.

However, the reliability of these adhesives has not been studied extensively. Partly because of the high initial variability on the mechanical performance of these joints. The aim of this research is to identify and correlate potential factors that have effect on the performance of an MS polymer joint. Both experimental and numerical studies are used to attain these results.

Both studies show that bond geometry has a marked effect on its mechanical performance. The experiments show that the assumption of a solid, uniform adhesive joint is invalid. However, if the size and location of defects in the bond line can be estimated, and an appropriate finite element model can be constructed, the joint performance can be estimated.
\end{abstract}

\section{KEYWORDS}

CONTACT K. Van Massenhove. Email: korneel.vanmassenhove@kuleuven.be 
Elastic adhesives; Butt joints; Complex modulus; MS polymer; Sealants; Novel adhesives

\section{Introduction}

Adhesive bonding is a major joining technique in various industrial and civil applications $[1,2]$. Among the many types of (semi) structural adhesives, viscoelastic adhesives have a relatively low modulus and strength, but are characterised by a large elongation at break [3, 4]. Applications include bonding of dissimilar materials and joints which require a structural, but flexible joint, for reasons of sealing, vibration damping or thermal expansion $[1,5,6]$.

To achieve the mentioned performance, these adhesives are typically used with thick bond lines. The viscoelastic, non-linear nature of these adhesives results in a strongly non-linear mechanical behaviour of these joint types $[7,8]$. In addition, it has been observed that their structural characteristics, like stiffness and strength show significant scatter [9].

The goal of this study is to identify and quantify the effect of multiple factors on the mechanical behaviour of viscoelastic butt joints. One of the important factors is the joint geometry, as it has been shown that bond line thickness has a significant effect on the elastic and fracture properties for brittle [10] and ductile [7, 11] adhesives. Other factors like imperfections in the adhesive also have a large effect. Examples of these are air pockets introduced in a viscous adhesive at application, or tears that appear in the bond during the hardening process of thick joints.

This work describes an experimental campaign which analyses the mechanical behaviour of a set of nominally identical butt joints.

The first and second part of this work discuss the set-up and results of experimental work on the adhesive joint dimensional and internal characteristics and the mechanical behaviour under static and dynamic loading conditions.

The third part describes how dedicated finite element models are developed and applied to (cor)relate heterogeneities in the adhesive joints to their observed stiffness and strength variability. 
The work summarises with conclusions and prospects to ongoing and future research in this area.

\section{Test coupons}

\subsection{Design}

According to Novatech International (Olen, Belgium), the manufacturer of Tec7, to achieve the optimal performance of the joint (tensile strength of at least 2.6 MPa, tear strength of 1.4 MPa), an adhesive thickness of $3 \mathrm{~mm}$ is recommended [12]. This large volume of adhesive allows for large deformation of the bond line. This increases the beneficial effects that the adhesive bond may introduce, such as an increased fracture toughness $[11,13]$. This large adhesive thickness provides gap-filling properties required for bonding large parts [14].

The detrimental effects of adhesive layer thickness in single lap shear tests, such as introduction of peel stresses and geometric non-linearities due to substrate bending [2], are unfortunately also increased. Moreover, the curing reaction of a one component MS polymer requires access ambient moisture [15]. Very wide bonds will decrease curing speed, while square or rectangular single lap joints may result in uneven curing.

Cylindrical butt joints do not have the disadvantages mentioned above, while still being relatively simple to manufacture. Butt joints can be used as tensile and torsion test geometries [16], while creep tests [17] can easily be performed. They also proven geometries for analytical [18-21], and solid and axisymmetric [22] finite element analysis methods.

Coupon geometry and nominal dimensions are shown in Figure 1a. The substrates are free cutting steel (material number 1.0715) cylinders to provide repetitive bonding quality. While the nominal dimensions can be set with the jig discussed in section 2.2 , some deviation of these nominal dimensions is possible and can be described by a few parameters, as shown in Figure 1b. This final coupon dimensioning is discussed in section 2.3 . 


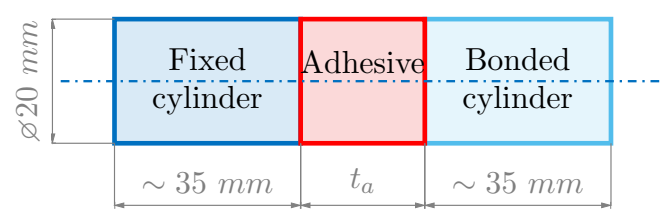

(a) Nominal butt joint coupon geometry and dimensions

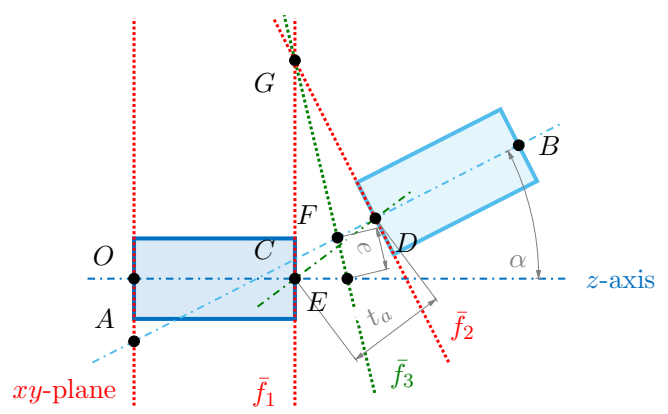

(b) Nominal and actual geometry of the butt joint coupons

Figure 1. Nominal and actual geometry of the butt joint coupons

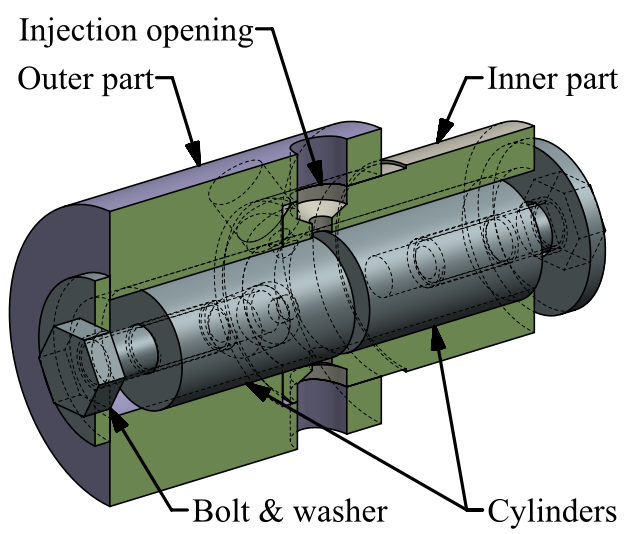

Figure 2. Sketch of the coupon production jig

\subsection{Sample manufacturing}

A set of twenty coupons with a nominal thickness of $3 \mathrm{~mm}$, is manufactured. The free cutting steel substrate cylinders are provided with an M8 threaded hole in the free end. This hole can be used to mount the coupons to test equipment, and is used to set the joint thickness in the PTFE bonding jig, shown in Figure 2.

Prior to bonding, the adhesion surface is faced on a lathe, lightly sanded to 800 grid, removing any surface oxidation. After cleaning with a 50/50 mixture of water and isopropanol, The substrates are inserted into the backs of the inner and outer part of the concentric jig cylinders. The substrate travel is set and limited by a fender washer and bolt. Once both jig halves are clamped together in a vice, the viscous adhesive is injected through one of the six radial holes, until the other five holes are 
Table 1. Geometric parameters and nominal values of the coupon dimensions

\begin{tabular}{lccl}
\hline Name & Symbol & Nominal value & Comments \\
\hline Coupon diameter & $d$ & $20 \mathrm{~mm}$ & Average diameter of both cylinder diameters \\
Adhesive thickness & $t_{a}$ & $3 \mathrm{~mm}$ & \\
Skew angle & $\alpha$ & $0^{\circ}$ & Angle between cylinder centrelines \\
Eccentricity & $e$ & $0 \mathrm{~mm}$ & $\begin{array}{l}\text { Distance between cylinder centrelines, } \\
\text { measured in the bisecting plane of the } \\
\text { cylinder top faces, }, \bar{f}_{3}\end{array}$ \\
& & & \\
\hline
\end{tabular}

filled. This set-up provides positive alignment of the substrates, while allowing large adhesive thicknesses and reducing the possibility of mixing air into the adhesive.

To ensure good bonding and complete hardening of the adhesive, the coupons are allowed to harden in the jig for one week and only tested after six weeks. One coupon was destroyed while removing it from the jig. The remaining nineteen samples were split in two sets. The first set of ten coupons are only subject to the quasi tensile test and the fracture surfaces were categorised as discussed in section 3.1. The second set underwent dynamic and quasi static tests, but the fracture surfaces are not studied further.

\subsection{Dimensioning}

The manufactured coupons are imperfect representations of the nominal geometry in Figure 1a. For further analysis, only a few dimensions of interest are used, as shown in Figure 1b and indicated in Table 1.

Using a Mitutoyo BHN 706 coordinate measuring machine, the location of points $O, A$ and $B$ can be determined. Combined with the lengths of both cylinders, measured before bonding, the actual dimensions of each adhesive layer can be calculated. The results of all nineteen tested coupons is shown in Figure 3.

Coupon diameter variability is quite low, with a coefficient of variance (COV) of less than one thousandth. Therefore, it is assumed that its effect is negligible. The other dimensions do have a significant spread, the adhesive has a COV of $12 \%$, while the angle and eccentricity have a COV of $54 \%$ and $43 \%$ respectively. Their effects are taken into account in the correlation analysis. 

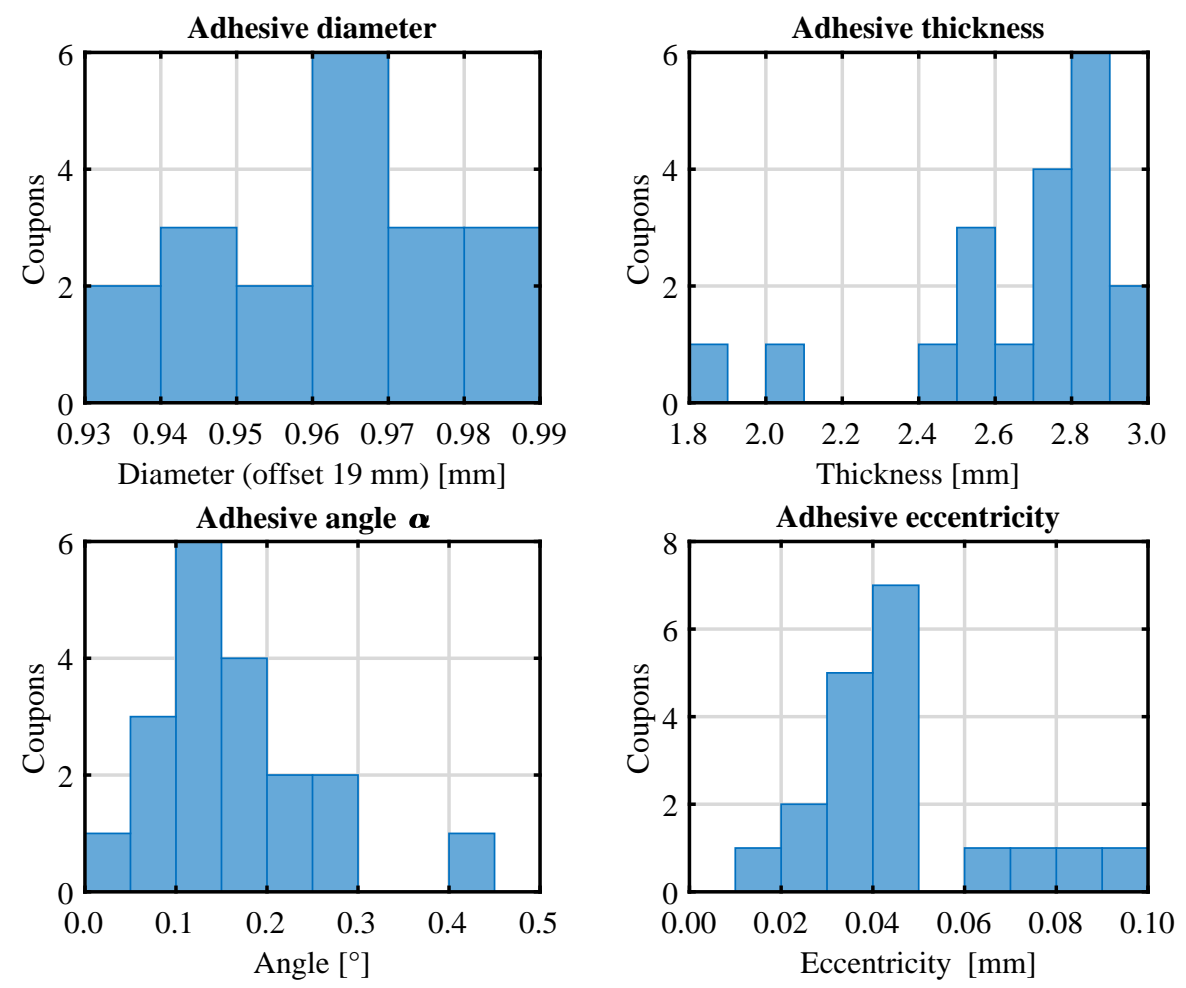

Figure 3. Histograms of coupon dimensions

\section{Test methods}

To characterise the mechanical behaviour of the butt joint test coupons, two test methods are used. The quasi static tensile test is conducted on all coupons, while the second batch of samples is also subjected to the dynamic test. If the dynamic test introduces some damage to the sample, that effect can be identified.

\subsection{Quasi static tensile test}

The quasi static test is a basic tensile test of the bonded coupons. Each is mounted in a Instron 3345 universal testing machine as shown in shown in Figure 4a. A crosshead displacement rate is set to result in a strain rate of $0.2 \%$ per second. For nominal coupon dimension, this results in a displacement rate of $0.36 \mathrm{~mm} / \mathrm{min}$. The apparent strain of the adhesive layer is based on the crosshead displacement corrected by the compliance of the test machine. This displacement is applied until the two coupon halves have completely separated. 


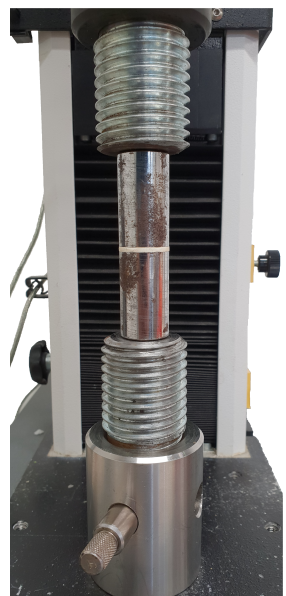

(a) Test set-up for the quasi static test

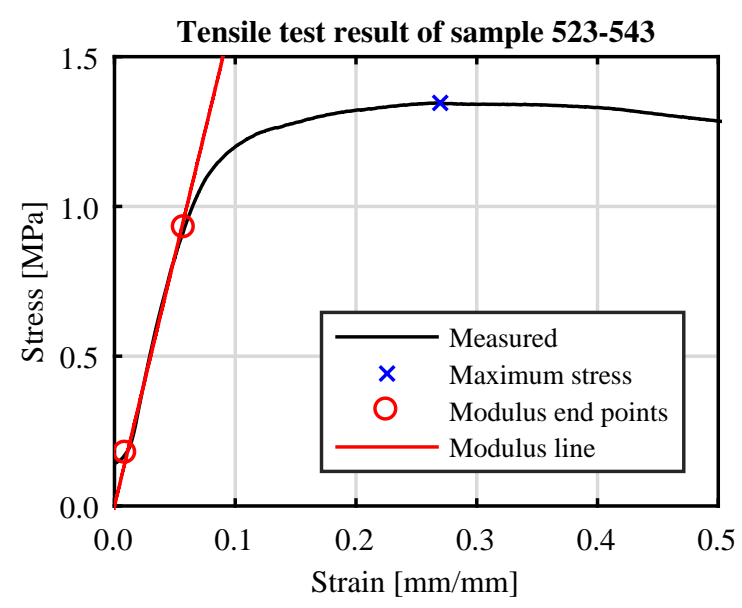

(b) Example tensile test result

Figure 4. Tensile test set-up and result

The experimental load-displacement data is converted into stress-strain data and condensed into three characteristic values. These are shown on a representative stressstrain curve in Figure 4b.

- Slope of the initial part of the stress-strain curve. The maximum deviation of this straight line off the stress-strain curve is limited to $2 \%$. This is the apparent modulus of the adhesive;

- Maximum applied stress, the joint strength;

- Strain at the maximum applied stress.

The failure surface of one coupon set was studied after test. The indicated zones are clearly different in colour or texture to the usually cohesive failure of the adhesive. Throughout this work, these areas are always indicated as a percentage of the total area of the section.

- Axial slits: zones where a clear, deep, but thin crevice is present in the adhesive.

- Air pockets: small to medium sized zones with a very smooth, hollow surface. These are the result of air bubbles that are trapped inside the adhesive during application.

- Adhesive failure: zones where no residue is left on the substrate. The adhesive has not sufficiently bonded to the steel. These zones may also be the result of an air bubble on the substrate surface, but this cannot be distinguished. 
- Interface failure: a thin residue is left on the substrate, which still shows through. These areas have a rough texture and often gradually change over into cohesive failure.

- Other: due to the production method, where the adhesive is injected radially through the butt joint, traces of the injection holes are still identifiable. Since these zones are visible, and may have an effect on the joint stiffness, these are taken into account for further analysis.

\subsection{Dynamic testing}

To estimate the stiffness of the adhesive at higher load frequencies, a dynamic test scheme is set up. As shown in Figure 5a, one end of the coupon is connected to the head of an electrodynamic shaker by a PCB TLD288D01 impedance head. The other end is loaded with the second accelerometer (Bruël\&Kjær 4514), mounting stud and a stack of washers to act as a variable mass load. Figure 5b shows the implementation of this setup. The system is excited with pseudo random noise at a low level to not excite any non-linearities.

This forms a single degree of freedom damped linear spring-mass system with base excitation. This set-up is preferred over the resonant and non-resonant methods proposed by Gade, Herlufsen, Zaveri and Konstantin-Hansen [23], as previous test have shown inconclusive results due to the required stiffness of the base.

The frequency response function (FRF) of the top acceleration $\left(a_{t}\right)$ to the acceleration of the bottom $\left(a_{b}\right)$ is measured using 500 averages in the frequency range of $0 \mathrm{~Hz}$ to $12.8 \mathrm{kHz}$ with a resolution of $2 \mathrm{~Hz}$. The theoretical FRF $\left(H_{1}\right)$ of the considered single degree of freedom system can be described by equation 1 and is frequency $(f$, or pulsation $\omega$ ) dependent.

$$
H_{1}(\omega)=\frac{a_{t}}{a_{b}}=\frac{k+c \omega \mathrm{j}}{-m \omega^{2}+k+c \omega \mathrm{j}}
$$

This FRF is determined by the equivalent spring $(k)$ and damping $(c)$ constants of the adhesive layer. These are in turn proportional to the apparent storage $\left(E^{\prime}\right)$ and loss modulus $\left(E^{\prime \prime}\right)$ of the adhesive, which in turn determine its (apparent) complex 


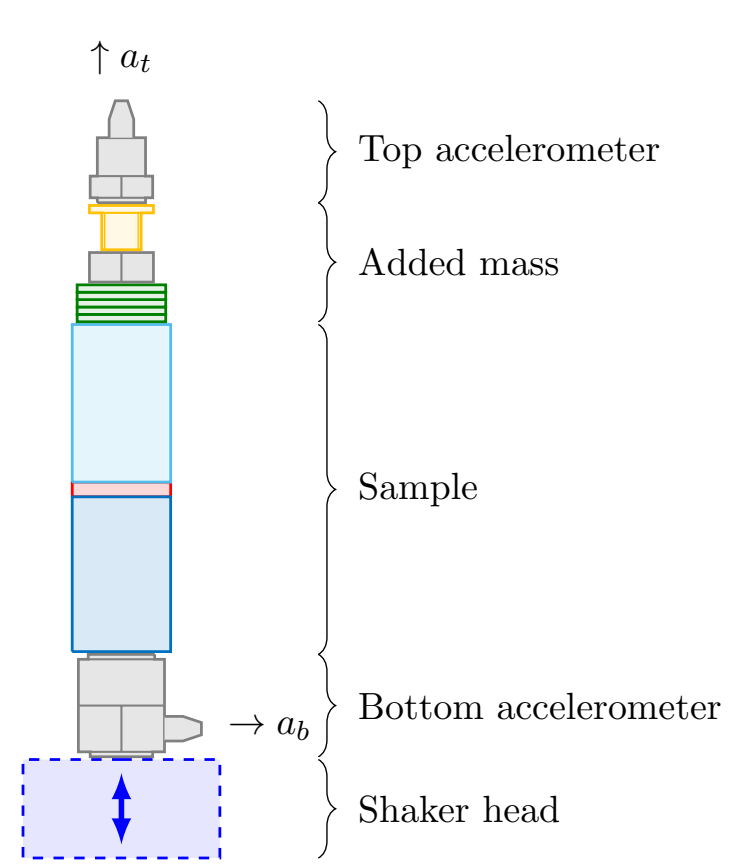

(a) Sketch of the dynamic test set-up

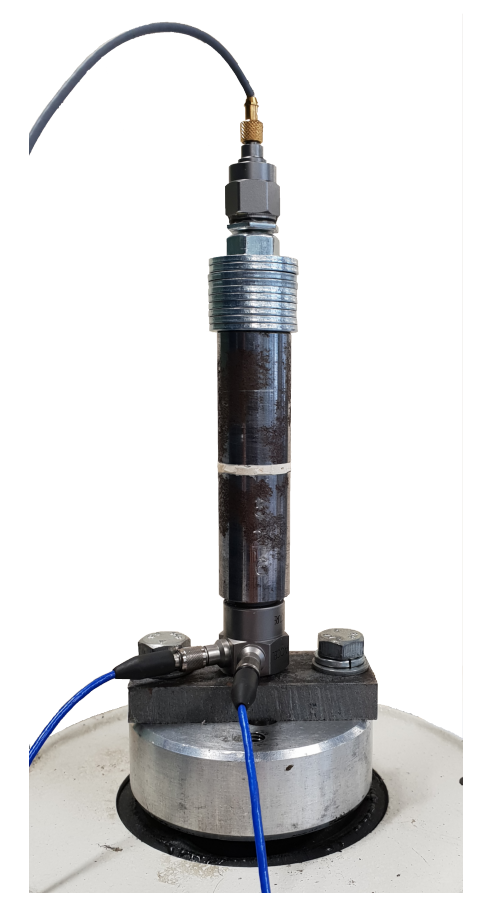

(b) Photo of the realised dynamic test set-up

Figure 5. Schematic representation and photo of the dynamic test set-up

modulus $E$. These relations are shown in equations 2 to 4 . The vibrating mass $m$ is the sum of half the sample mass and the added mass loading. The adhesive mass is considered negligible, as it is about 2 gram of the total sample mass of about 154 gram, this results in an error of the estimated vibrating sample mass of less than $0.5 \%$.

$$
\begin{aligned}
& E^{\prime}=\frac{4 k t_{a}}{\pi d^{2}} \\
& E^{\prime \prime}=\frac{4 c t_{a}}{\pi d^{2}} \\
& E=\sqrt{E^{\prime 2}+E^{\prime \prime 2}}
\end{aligned}
$$

\section{Experimental results}

Once the coupons' adhesive is thoroughly hardened, the two sample sets can be subjected to the tests as planned above. Section 4.1 describes the test results for the first set of ten, while section 4.2 discusses the test results of the second set. 
Table 2. Overview of the first coupon set test results

\begin{tabular}{lcccl} 
Quantity & Minimum & Median & Maximum & Unit \\
\hline Joint Thickness & 2.6 & 2.8 & 2.9 & $\mathrm{~mm}$ \\
Apparent modulus & 11.4 & 14.1 & 16.7 & $\mathrm{MPa}$ \\
Maximum stress & 1.3 & 1.4 & 1.4 & $\mathrm{MPa}$ \\
Strain & 26.7 & 32.5 & 39.0 & $\%$ \\
Air pocket area & 0.0 & 2.0 & 4.8 & $\%$ \\
Adhesive failure area & 0.0 & 0.7 & 2.4 & $\%$ \\
Interface failure area & 5.3 & 10.1 & 30.2 & $\%$ \\
Other failure area & 1.4 & 3.0 & 6.7 & $\%$
\end{tabular}

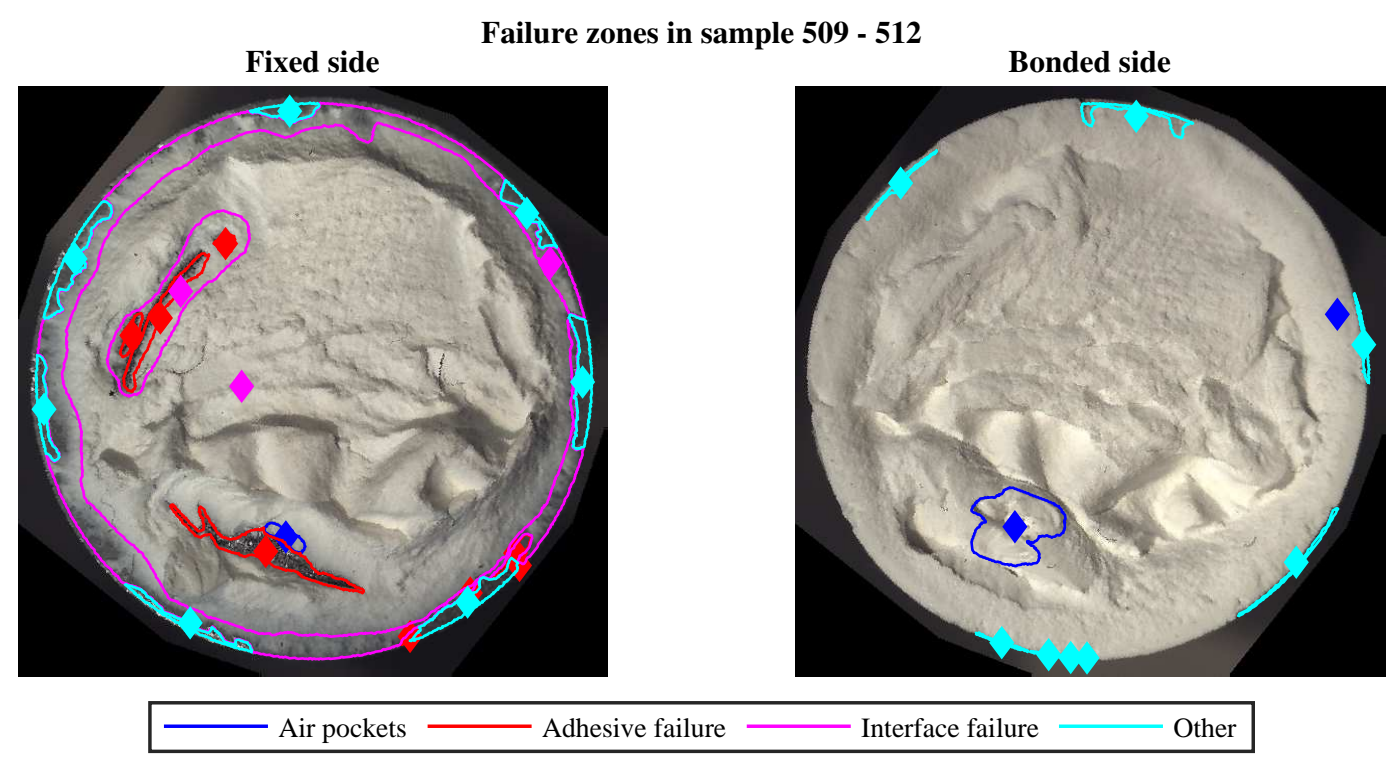

Figure 6. Example of the identified failure zones after a destructive tensile test, the diamonds indicate the zone centroid

\subsection{Geometry, damage and mechanical performance}

The results of the tensile tests are similar to those shown in Figure $4 \mathrm{~b}$. The cross section of both coupon halves, with the categorised failure zones is shown in Figure 6 . Table 2 summarises the results of these tensile tests.

It is to be expected that coupon geometry and possible failure area ratios have an effect on the mechanical properties of the joint. To estimate the validity of this statement, a correlation analysis is performed on the results. Figure 7 shows the resulting correlation and probability. A low $p$-value indicates a low probability that corresponding correlation can be discarded as a coincidence, also indicated by the high absolute value of the correlation.

It is clear that there is some correlation between the coupon geometry and its mechanical performance. Especially the sample eccentricity, which probably has a beneficial effect on the sample stiffness $(+0.64)$ and may have a small influence on the 


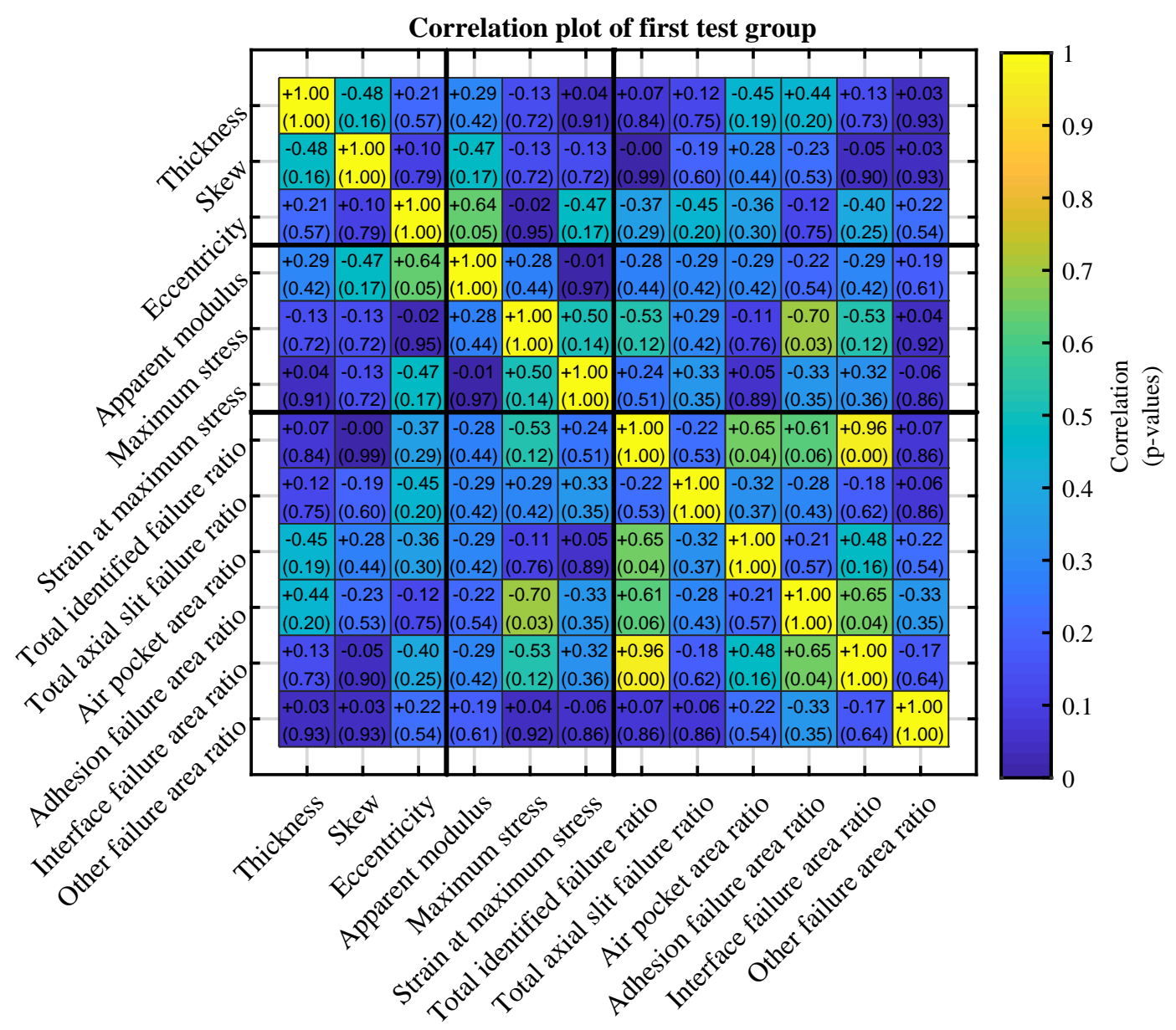

Figure 7. Correlation plot of the first set of coupons, correlating geometry, mechanical properties and failure area ratios 
strain at maximum stress.

The results also show that the apparent modulus is seemingly not linked to the stress a joint can receive before inadvertently failing and neither to the strain at that instant. However, the joint strength and strain do show some limited $(+0.50)$ correlation.

The adhesive strength does seem to be mainly correlated $(-0.70)$ to the amount of substrate area that has not bonded to the adhesive. This is to be expected: a large area of unbonded substrate must be detrimental to joint strength. However, it appears that a lower bonded area does neither affect sample stiffness, nor strain at maximum strength. The section in Figure 6 also shows that interface failure may be initialized by a lack of adhesion nearby. This is also shown by the correlation of these defect types $(+0.65)$ and a weak correlation of the interface failure ratio to the sample strength $(-0.53)$. This correlation is probably not due to the lower strength of the adhesive in these zones, as this is still a form of cohesive failure.

While the amount of air pockets contributes to the total area occupied by defects $(+0.65)$, it does not seem that these have any effect on the mechanical performance of a joint. This may be due to the relatively small area of each individual pocket, compared to the large area an adhesive failure takes up. A small defect in the bulk adhesive will influence the mechanical performance of a joint less than a large one at the joint interface.

\subsection{Geometry, dynamic mechanical performance}

The set-up shown in Figure 5 is used to identify the apparent complex modulus of the adhesive in nine coupons. As with the first set, a correlation analysis will be performed to estimate the relation of the adhesive complex modulus at high frequency to its apparent modulus in a quasi-static environment. A typical measured FRF of a dynamic test is shown in Figure 8a.

The result of all dynamic tests is shown in Figure 8b. For each coupon, a straight line is fitted through the measured apparent complex moduli at each resonance frequency and the quasi static apparent modulus, with both datasets having the same weight. This fitted line can be used to estimate the apparent modulus at $0 \mathrm{~Hz}$ and 


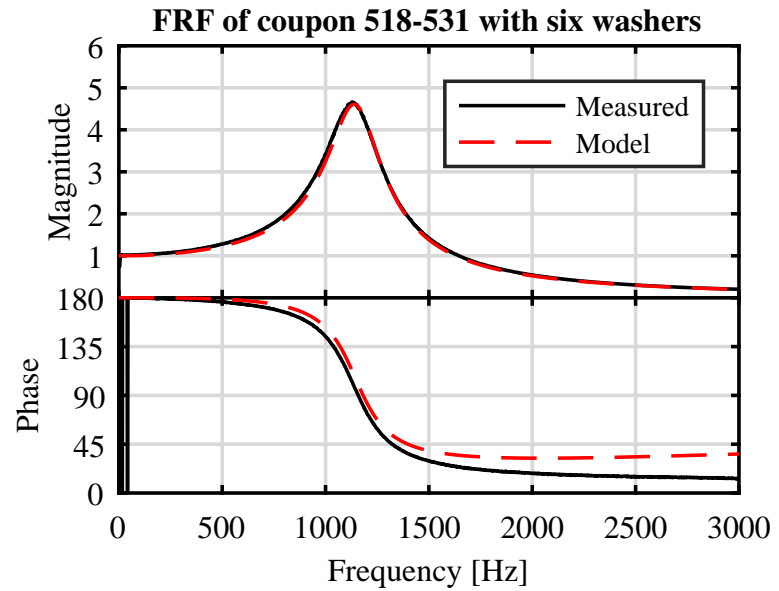

$t_{a}=2.77 \mathrm{~mm}, m=139.8$ gram, $f_{r}=1126 \mathrm{~Hz}$

$k=7.3 \mathrm{MN} / \mathrm{m}, c=226 \mathrm{~N} \mathrm{~s} / \mathrm{m}$

$E=64.8 \mathrm{MPa}, E^{\prime \prime}=2 \mathrm{kPa}$

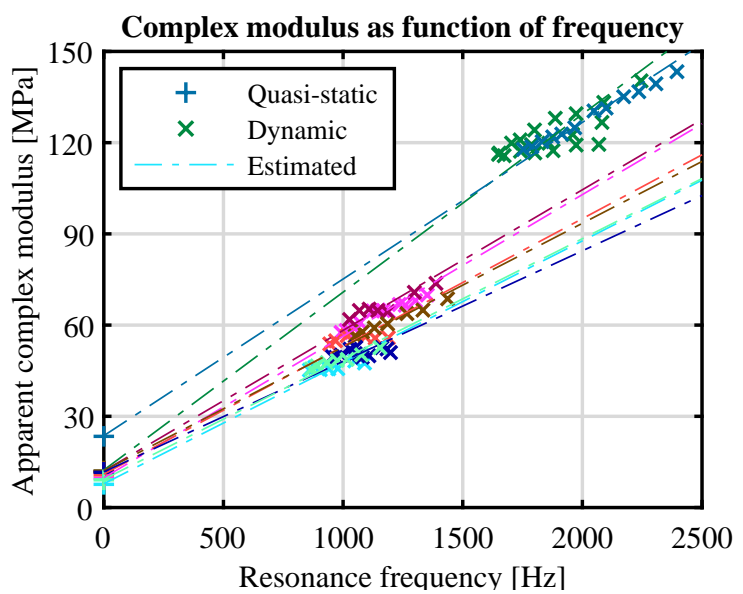

(b) Apparent complex modulus and damping ratio as function of frequency

(a) Example of the measured and modelled FRF of a sample

Figure 8. Detail of one dynamic test and the result of all test

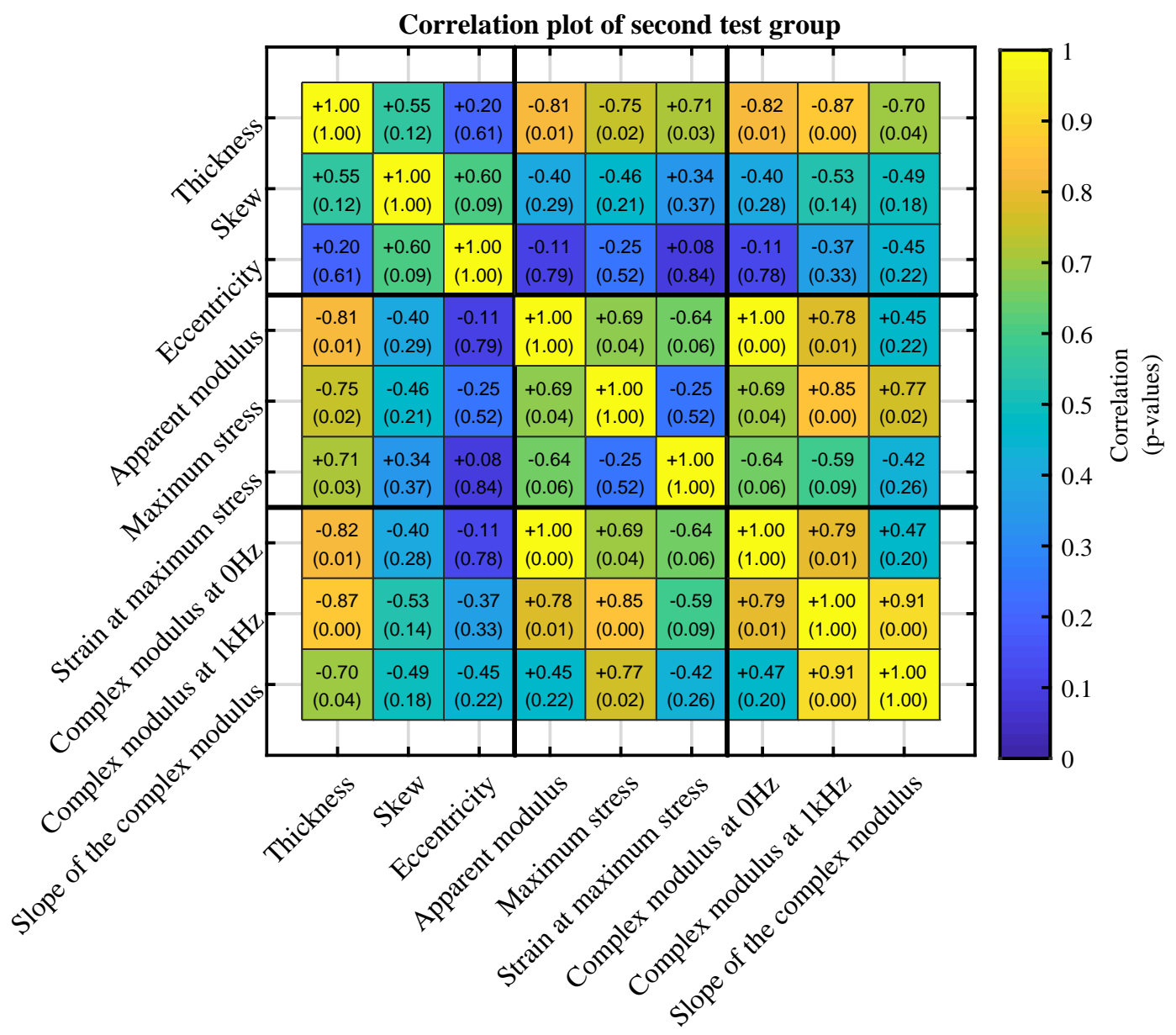

Figure 9. Correlation plot of the second set of coupons, correlating geometry, quasi static and dynamic mechanical properties of the joints 
$1 \mathrm{kHz}$, as it is clear that determining one definitive complex for all frequencies is impossible. Also, the slope of this line may contain valuable information. Table 3 summarises these results. As with the previous coupon set, the correlation between these results is calculated and shown in Figure 9.

Unlike the first set, the coupon dimensions are more clearly correlated. However, the coupons in this set show a markedly higher variability of the adhesive thickness $((2.5 \pm 0.9) \mathrm{mm})$ compared to the first set $((2.8 \pm 0.3) \mathrm{mm})$. This increased range increases the sensitivity to small changes in sample skew or eccentricity. The correlation between coupon skew and eccentricity is partly due to the definition of the eccentricity, as shown in FIG.1b, which means that an increase in skew angle offsets both cylinder axes in plane $\bar{f}_{3}$.

Since the range of measured adhesive layer thicknesses has increased, its effect is clearly represented by a large correlation between it and the joint mechanical parameters, both at low and high frequencies. A decrease of adhesive thickness increases its stiffness and strength, but decreases its ability to absorb any applied deformation. As discussed in literature $[7,9,11,13,22,24]$ and in chapter 5 , the (stiff) substrate does not allow any radial deformation of the sample. In a thin adhesive layer, this leads to a volumetric loading of the adhesive, rather than tensile and/or shear. Since the rubber like adhesive is nearly incompressible, its bulk modulus is very high compared to its tensile or shear modulus. A decreased joint thickness will therefore increase the volumetric loading of the adhesive, increasing its stiffness.

The correlation between skew angle and eccentricity to the mechanical properties is much lower than the correlation between thickness and mechanical performance. This correlation is likely fully attributable to the correlation between dimensioning parameters, rather than a direct relation.

Table 3. Overview of the second coupon set test results

\begin{tabular}{lcccl}
\hline Quantity & Minimum & Median & Maximum & Unit \\
\hline Joint Thickness & 1.8 & 2.8 & 2.9 & $\mathrm{~mm}$ \\
Apparent modulus & 7.6 & 11.6 & 23.4 & $\mathrm{MPa}$ \\
Maximum stress & 1.2 & 1.3 & 1.4 & $\mathrm{MPa}$ \\
Strain & 28.6 & 41.6 & 51.2 & $\%$ \\
Complex modulus at $0 \mathrm{~Hz}$ & 7.8 & 11.7 & 23.6 & $\mathrm{MPa}$ \\
Complex modulus at $1 \mathrm{kHz}$ & 47.8 & 53.0 & 75.2 & $\mathrm{MPa}$ \\
Complex modulus slope & 36.3 & 42.0 & 58.5 & $\mathrm{kPa} / \mathrm{Hz}$ \\
\hline
\end{tabular}


The linear regression of the measured apparent modulus is weighted, so that the quasi static measurement has the same weight as the apparent modulus measured with the dynamic set-up. This explains the unity correlation of the estimated complex modulus at zero Hertz to the one measured with the quasi static method. However, this correlation decreases slightly at higher $1 \mathrm{kHz}$, but still remains high at +0.78 .

This regression analysis method also explains the correlation between the maximum stress and strain at maximum stress on one hand and the moduli measured with the dynamic method. However, the high correlation between maximum stress and the slope of the apparent complex modulus line $(+0.77)$ cannot. It may be explained by the relation between adhesive thickness and its effect on both strength and slope of the modulus line.

\section{Finite element analysis}

The samples discussed in chapter 4 are not perfect, it is therefore not possible to identify the effect of a single change in sample geometry, or any other discussed parameter. Modelling the joint may aid in understanding these effects. An analytical method, as discussed by Sawa [21] can aid in the initial analysis, but does not support nonlinear behaviour of the adhesive. A nonlinear axisymmetric finite element method, such as performed by Adams, Coppendale and Peppiatt [22] can provide valuable information. A disadvantage of axisymmetric modelling is that local defects such as shown in Figure 6 cannot be added. Solid 3D elements do not show this disadvantage, so a set of nonlinear 3D finite element analyses will be performed. These are based on the Siemens NX Nastran 12 solution 601-106 solver.

\subsection{Material model}

The non-linear behaviour of the coupon test shown in Figure $4 \mathrm{~b}$ is not merely due to the coupon geometry. Tensile tests on adhesive films have shown a clear nonlinear, rubberlike behaviour of the adhesive. A suitable, yet incompressible, material model to represent this behaviour is the hyperelastic Ogden model [25, 26]. This material model has been shown to accurately model the behaviour of rubberlike solids [27-29]. 
NX Nastran provides a slightly modified model that includes material compressibility [30]. Its constitutive model is shown in equation 6 , where $\lambda_{k}$ is the material stretch in the $k$-th direction. The strain energy density function $w$ can be split into a distortion $w_{D}$ and volumetric $w_{V}$ part. The distortion energy density function is of the $N$-th order, while the volumetric function is only of first order.

$$
\begin{aligned}
w(\lambda) & =w_{D}+w_{V} \\
& =\sum_{i=1}^{N} \frac{\mu_{i}}{\alpha_{i}}\left(\bar{\lambda}_{1}^{\alpha_{i}}+\bar{\lambda}_{2}^{\alpha_{i}}+\bar{\lambda}_{3}^{\alpha_{i}}-3\right)+\frac{1}{2} K(J-1)^{2} \\
J & =\lambda_{1} \lambda_{2} \lambda_{3} \\
\bar{\lambda}_{k} & =\frac{\lambda_{k}}{J^{\frac{1}{3}}}
\end{aligned}
$$

The deformation energy density function has $\mu_{i}$ and $\alpha_{i}$ as model parameters, while the volumetric energy density function is only dependent on an the material bulk modulus $K$. If only a uni-axial tensile test is performed, the bulk modulus cannot be determined, but if the material Poisson coefficient $\nu$ is known, initial Young's $\left(E_{0}\right)$, shear $\left(G_{0}\right)$ and bulk $\left(K_{0}\right)$ modulus can be calculated by equations 9 to 11 :

$$
\begin{aligned}
& E_{0}=(1+\nu) \sum_{i=1}^{N} \mu_{i} \alpha_{i} \\
& G_{0}=\frac{1}{2} \sum_{i=1}^{N} \mu_{i} \alpha_{i} \\
& K_{0}=\frac{1+\nu}{3(1-2 \nu)} \sum_{i=1}^{N} \mu_{i} \alpha_{i}
\end{aligned}
$$

A third order model is fitted to a set of uni-axial stress-strain curves of the same adhesive, but of a different batch. The model parameters are shown in Table 4. No failure, thermal or viscoelastic material properties are defined in the model. Therefore, these simulations are only valid in a limited domain, before any adhesive failure occurs. 
Table 4. Ogden material model parameters and initial moduli

\begin{tabular}{ccc}
\hline$\alpha$ & $\mu$ & $\nu$ \\
\hline 0.049 & $61 \mathrm{MPa}$ & \\
0.54 & $2.2 \mathrm{MPa}$ & 0.499 \\
4.1 & $0.055 \mathrm{MPa}$ & \\
\hline$E_{0}$ & $G_{0}$ & $K_{0}$ \\
$6.6 \mathrm{MPa}$ & $2.2 \mathrm{MPa}$ & $1099 \mathrm{MPa}$ \\
\hline
\end{tabular}

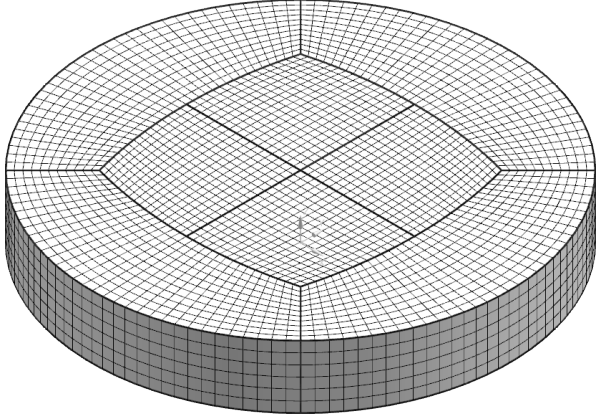

(a) Finite element model of the adhesive layer

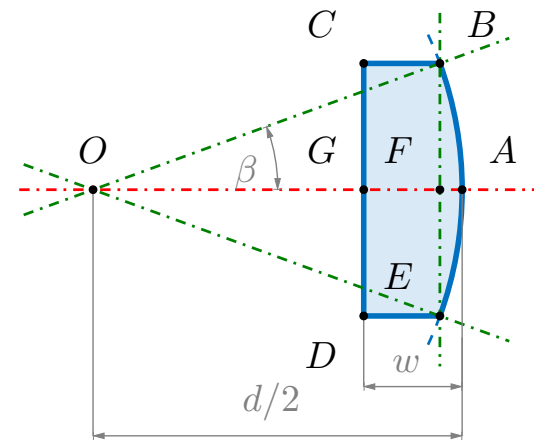

(b) Generalised geometry of the 'other' failure type

Figure 10. Finite element model and detail of adhesive defect model

\subsection{Geometry}

The finite element model is a 3D solid brick element (CHEXA20) model of the adhesive only. The steel substrates are not modelled, as ratio of the stiffness of the steel compared to that of the adhesive is quite high. The circular faces are modelled according to the method proposed by Štigler [31] with 128 elements on the bond face circumference. This method allows rectangular elements on circular faces while keeping element distortion low. Six layers of elements were used for all simulations. The model is dimensioned according to the nominal adhesive coupon. The meshed structure is shown in Figure 10a

The bottom surface of the adhesive is fully fixed, while a time dependent displacement is enforced on the top face. The total applied load along the cylinder axis is taken as the output of these simulations. As with the experiments, an apparent stress-stain curve is constructed, based on these results. 


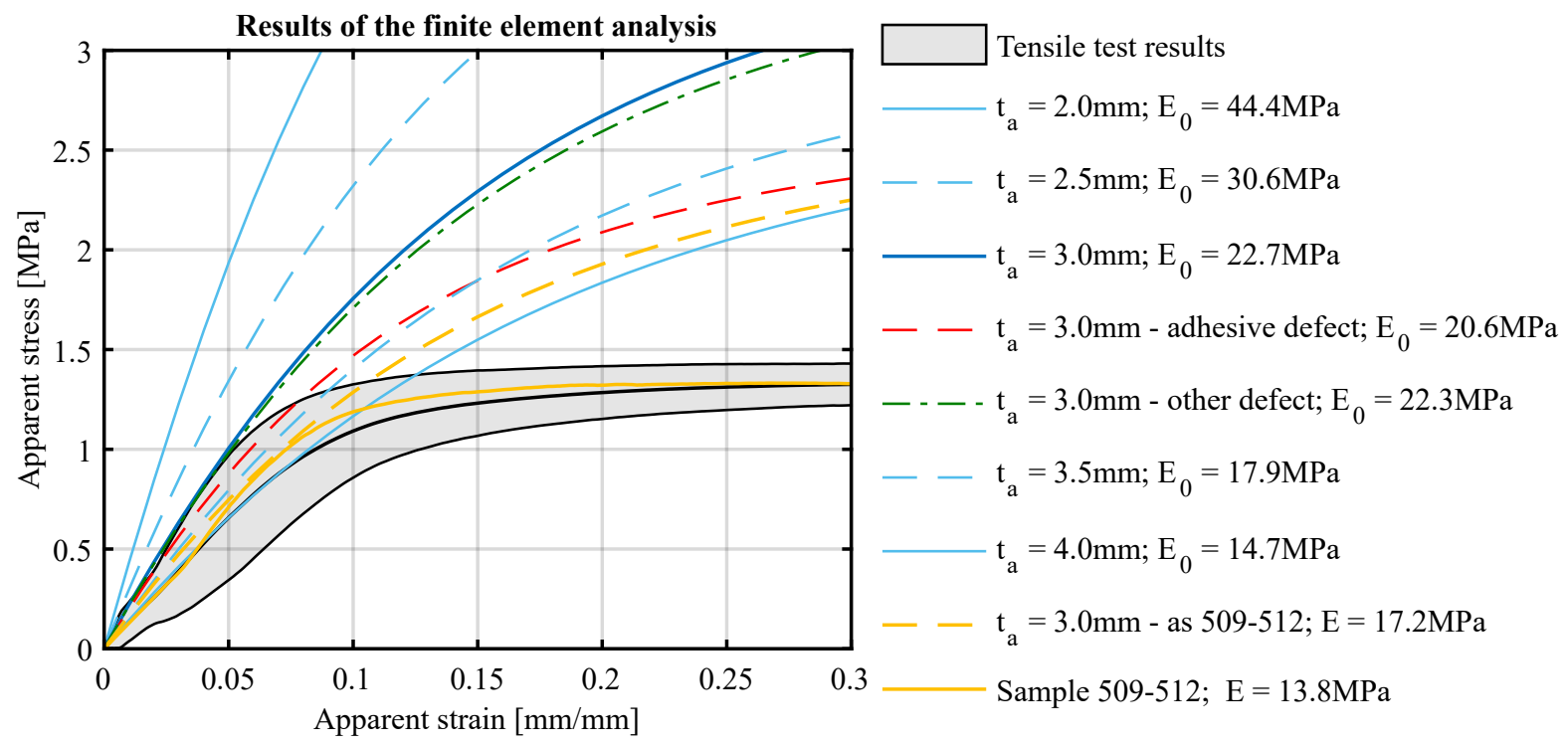

Figure 11. Results of the coupon tensile tests and finite elements analysis of the adhesive layer

\subsection{Numeric results}

The main goal is to estimate the effect of adhesive layer thickness on the apparent modulus of the joint. The modelled geometry also allows the introduction of a large adhesion failure zone in one of the central quadrants of the top or bottom surface mesh. A zone similar in size, repetition and shape of the type "Other" in Figure 6 is added to the model. Its shape is shown in Figure 10b. The angle $\beta$ is $8.9^{\circ}$, and the shape's with $(w)$ is $0.66 \mathrm{~mm}$. This zone is repeated each six times on both sides of the adhesive. There are no constraints applied in al twelve zones, to represent a completely debonded adhesive.

Figure 11 shows the tensile test data range as the grey zone. The mean behaviour of the sample is indicated by the central solid black line. The erratic behaviour at low strains is due to the clamping method.

Next to the measurement data, the simulated apparent stress-strain curves for coupons with an adhesive thickness of $1.5 \mathrm{~mm}$ to $4.0 \mathrm{~mm}$ are shown. For low thicknesses, these show a markedly higher apparent modulus than the experiments, even the coupons with low adhesive thicknesses do not have a similarly elevated apparent modulus. This may be due to a difference in the performance of adhesive batches, as previous experiments have shown a higher similarity. 
Similar to the experimental results, the joint stiffness is dependent on adhesive thickness, even more so than experimentally shown. This effect decreases with thickness, showing a hyperbolic dependence of the apparent modulus on adhesive thickness.

At about $7.5 \%$ strain, the measurement data starts to diverge from the finite element results. This indicates the onset of bond line failure. The coupons show high toughness, as the joint can still be loaded significantly, while allowing further deformation before ductile failure. As element rupture or adhesive failure is not modelled, the simulated apparent stress-strain curve shows continuously increasing apparent stress.

The analysis results for bonds with adhesive defects, and a bond line thickness of $3 \mathrm{~mm}$, show the effect of small adhesive failures at the circumference and a large defect in the adhesive centre. Small debonds have little effect on sample stiffness, as indicated by the small correlation in Figure 7. Very large defects show a marked decrease of sample stiffness and attainable strength, as the simulated curve shows a lower apparent modulus at the start and a decreased slope at higher strains.

As a final modelling experiment, the defects shown in the section in Figure 6 are implemented in the $3 \mathrm{~mm}$ thick model. These adhesive defects are modelled by removing the elements located inside one of the indicated failure zones. The experimental and simulated tensile test result are shown in Figure 11 as the orange solid and dashed lines. At an apparent strain of $7.5 \%$, the coupon starts to fail. While this failure is not modelled, the maximum principal stress in the core of the adhesive is about $3.8 \mathrm{MPa}$, just above the $3.5 \mathrm{MPa}$ stress limit according to a tensile test. The apparent modulus of the coupon is slightly lower than simulated, but this can be attributed to several factors. These include the slightly lower coupon adhesive thickness, of $2.82 \mathrm{~mm}$, different calculation method and potential error of the Ogden model parameter estimation.

\section{Conclusion}

Performance of these MS polymer adhesives is highly dependent on bond dimensions. Small perturbations can lead to large changes in joint performance, as shown in the experiments above. However, these effects can be quantified and related. This in turn allows for the estimation of design tolerances. If it can be assured that no large bond 
defects are present, a joint performance range can be estimated. If potential defects are unavoidable, this estimated range must be increased, as both joint stiffness and strength can decrease considerably.

The Ogden material model shows good performance for the considered finite element simulations, but the model parameters must be adequately estimated. The discrepancy between simulated joint performance and experimental results show that batch-to-batch variation may be considerable. Correct performance estimation requires small batch variability or a quite time consuming experimental campaign for each batch.

The solid finite element model, where localised defects in the adhesive are modelled, shows promise as an analysis method. It allows the estimation of the effect single or multiple adhesive defects have on the elastic behaviour of a joint. For a hyperelastic material, such as MS polymers, the Ogden material model shows good performance with adequately estimated model parameters. Estimation of initial joint failure is possible, but the exact failure mode and the evolution of the joint performance cannot be assessed with the current setup. However, further study of this analysis strategy may provide useful information for industrial bonding applications where bond line defects may be unavoidable.

\section{Acknowledgement(s)}

This research was made possible by the funding and time provided by KU Leuven.

\section{References}

[1] Lucas Filipe Martins da Silva, Andreas Öchsner, and Robert D. Adams, editors, Handbook of Adhesion Technology volume 2, Springer Berlin, Heidelberg (2011).

[2] L. D. R. Grant, Robert D. Adams, and Lucas Filipe Martins da Silva, "Experimental and numerical analysis of single-lap joints for the automotive industry", International Journal of Adhesion and Adhesives 29(4), pp. 405-413 (2009).

[3] Jingxin Na, Yu Liu, Yuanwu Wang, Le Pan, and Yakun Yan, "Effect of Tem- 
perature on the Joints Strength of an Automotive Polyurethane Adhesive", The Journal of Adhesion 92(1), pp. 52-64 (2016).

[4] Jingxin Na, Yu Liu, Yisa Fan, Wenlong Mu, Xin Chen, and Yakun Yan, "Effect of temperature on the joint strength of a silyl-modified polymer-based adhesive", The Journal of Adhesion 93(8), pp. 626-639 (2017).

[5] Firas Awaja, Michael Gilbert, Georgina Kelly, Bronwyn Fox, and Paul J. Pigram, "Adhesion of polymers", Progress in Polymer Science (Oxford) 34(9), pp. 948968 (2009).

[6] Adnan Ozel, Betul Yazici, Salih Akpinar, Murat Demir Aydin, and Şemsettin Temiz, "A study on the strength of adhesively bonded joints with different adherends", Composites Part B: Engineering 62, pp. 167-174 (2014).

[7] Raul D. S. G. Campilho, D. C. Moura, Mariana D. Banea, and Lucas Filipe Martins da Silva, "Adhesive thickness effects of a ductile adhesive by optical measurement techniques", International Journal of Adhesion and Adhesives 57, pp. 125-132 (2015).

[8] M. Y. Tsai and J. Morton, "An experimental investigation of nonlinear deformations in single-lap joints", Mechanics of Materials 20(3), pp. 183-194 (1995).

[9] José M. Arenas, Julián J. Narbón, and Cristina Alía, "Optimum adhesive thickness in structural adhesives joints using statistical techniques based on Weibull distribution", International Journal of Adhesion and Adhesives 30(3), pp. 160165 (2010).

[10] Davide Castagnetti, A. Spaggiari, and E. Dragoni, "Effect of bondline thickness on the static strength of structural adhesives under nearly-homogeneous shear stresses", Journal of Adhesion 87(7-8), pp. 780-803 (2011).

[11] Mariana D. Banea, Lucas Filipe Martins da Silva, and Raul D. S. G. Campilho, "The effect of adhesive thickness on the mechanical behavior of a structural polyurethane adhesive", Journal of Adhesion 91(5), pp. 331-346 (2014).

[12] Novatech International, "Tec7 Technical fiche" (2016).

[13] J. C. P. Figueiredo, Raul D. S. G. Campilho, E. A. S. Marques, J. J. M. Machado, and Lucas Filipe Martins da Silva, "Adhesive thickness influence on the shear fracture toughness measurements of adhesive joints", International Journal of 
Adhesion and Adhesives 83(March), pp. 15-23 (2018).

[14] Garbiñe Fernandez, Dirk Vandepitte, Hodei Usabiaga, and Stijn Debruyne, "Static and cyclic strength properties of brittle adhesives with porosity", International Journal of Fatigue 117(August), pp. 340-351 (2018).

[15] Edward M. Petrie, Handbook of adhesives and sealants volume 1 of McGraw-Hill Handbooks, McGraw-Hill Education 2 edition (2007).

[16] N. Arnaud, R. Créac'Hcadec, and J. Y. Cognard, "A tension/compressiontorsion test suited to analyze the mechanical behaviour of adhesives under nonproportional loadings", International Journal of Adhesion and Adhesives 53, pp. 3-14 (2014).

[17] Binti Ramli Mizah, Yu Sekiguchi, and Chiaki Sato, "Novel method to measure the creep strength of adhesively bonded butt joints subjected to constant loading using a hydro-pneumatic testing machine", Journal of Adhesion 92(1), pp. 65-79 (2016).

[18] Lucas Filipe Martins da Silva, Paulo J. C. das Neves, Robert D. Adams, and J. K. Spelt, "Analytical models of adhesively bonded joints-Part I: Literature survey", International Journal of Adhesion and Adhesives 29(3), pp. 319-330 (2009).

[19] Lucas Filipe Martins da Silva, Paulo J. C. das Neves, Robert D. Adams, A. Wang, and J. K. Spelt, "Analytical models of adhesively bonded joints-Part II: Comparative study", International Journal of .. 29(3), pp. 331-341 (2009).

[20] T. Sawa, K. Temma, and Y. Tsunoda, "Axisymmetric stress analysis of adhesive butt joints of dissimilar solid cylinders subjected to external tensile loads", International Journal of Adhesion and Adhesives 9(3), pp. 161-169 (1989).

[21] Toshiyuki Sawa, "Analytical models with stress functions", In Lucas Filipe Martins da Silva and Andreas Öchsner, editors, Modeling of Adhesively Bonded Joints chapter 3, , pp. 53-92. Springer Berlin, Heidelberg (2008).

[22] Robert D. Adams, J. Coppendale, and N. A. Peppiatt, "Stress analysis of axisymmetric butt joints loaded in torsion and tension", The Journal of Strain Analysis for Engineering Design 13(1), pp. 1-10 (1978).

[23] Svend Gade, Henrik Herlufsen, K Zaveri, and H Konstantin-Hansen, "Complex Modulus and Damping Measurements using Resonant and Non-resonant Meth- 
ods", Brüel 83 Kjaer Technical Review 2(2), pp. 28-44 (1994).

[24] A. Akhavan-Safar, M. R. Ayatollahi, and Lucas Filipe Martins da Silva, "Strength prediction of adhesively bonded single lap joints with different bondline thicknesses: A critical longitudinal strain approach", International Journal of Solids and Structures 109, pp. 189-198 (2017).

[25] Ray W. Ogden, "Large deformation isotropic elasticity -on the correlation of theory and experiment for incompressible rubberlike solids", Proceedings of the Royal Society of London A: Mathematical, Physical and Engineering Sciences 326(1567), pp. 565-584 (1972).

[26] Ray W. Ogden, Non-Linear Elastic Deformations, Dover Civil and Mechanical Engineering. Dover Publications Mineola, New York (1997).

[27] Ray W. Ogden, "Recent Advances in the Phenomenological Theory of Rubber Elasticity", Rubber Chemistry and Technology 59(3), pp. 361-383 (1986).

[28] A. Benjeddou, E. Jankovich, and T. Hadhri, "Determination of the Parameters of Ogden's Law Using Biaxial Data and Levenberg-Marquardt- Fletcher Algorithm", Journal of Elastomers 83 Plastics 25(3), pp. 224-248 (1993).

[29] G. Marckmann and E. Verron, "Comparison of Hyperelastic Models for RubberLike Materials", Rubber Chemistry and Technology 79(5), pp. 835-858 (2006).

[30] Siemens Product Lifecycle Management Software Inc., NX Nastran 12 Quick Reference Guide, Siemens PLM Software Plano, Texas (2017).

[31] Jaroslav Štigler, "Optimal Mapped Mesh on the Circle", In Konference ANSYS 2009 , pp. 245-252 Pilsen (2009). TechSoft Engineering. 